\title{
IEEE 1588 Time Synchronisation Performance for IEC 61850 Transmission Substations
}

DOI:

10.1016/j.ijepes.2018.11.036

\section{Document Version}

Accepted author manuscript

Link to publication record in Manchester Research Explorer

\section{Citation for published version (APA):}

Han, M., Guo, H., \& Crossley, P. (2018). IEEE 1588 Time Synchronisation Performance for IEC 61850

Transmission Substations. International Journal of Electrical Power \& Energy Systems, 107, 264-272.

https://doi.org/10.1016/j.ijepes.2018.11.036

\section{Published in:}

International Journal of Electrical Power \& Energy Systems

\section{Citing this paper}

Please note that where the full-text provided on Manchester Research Explorer is the Author Accepted Manuscript or Proof version this may differ from the final Published version. If citing, it is advised that you check and use the publisher's definitive version.

\section{General rights}

Copyright and moral rights for the publications made accessible in the Research Explorer are retained by the authors and/or other copyright owners and it is a condition of accessing publications that users recognise and abide by the legal requirements associated with these rights.

\section{Takedown policy}

If you believe that this document breaches copyright please refer to the University of Manchester's Takedown Procedures [http://man.ac.uk/04Y6Bo] or contact uml.scholarlycommunications@manchester.ac.uk providing relevant details, so we can investigate your claim.

\section{OPEN ACCESS}




\title{
IEEE 1588 Time Synchronisation Performance for IEC 61850 Transmission Substations
}

\author{
Mingyu $\mathrm{Han}^{1 *}$, Hao Guo ${ }^{2}$, Peter Crossley ${ }^{1}$
}

\footnotetext{
${ }^{1}$ School of Electrical and Electronic Engineering, The University of Manchester, Manchester, M13 9PL, UK

${ }^{2}$ The Power Networks Demonstration Centre, University of Strathclyde, Glasgow, G68 0EF, UK.

"Corresponding author. Email: mingyu.han@manchester.ac.uk
}

\begin{abstract}
Accurate timestamping with $1 \mu$ s precision is required by power system monitoring and control equipment, including: Phase Measurement Units (PMU) for real-time measurement and control; travelling wave detectors for fault location, protection and control; and sample value measurement techniques associated with the IEC 61850 process bus. The conventional way of achieving this is to transmit 1-PPS or IRIG-B timing signals using dedicated cables but such an approach is expensive and difficult to update as secondary control systems evolve. IEEE 1588 precision time protocol (PTP) allows the transmission of time information with submicrosecond accuracy in an Ethernet network. However, a comprehensive study is required to enhance the confidence of the power industry in using PTP in present and future IEC 61850 based substations. This paper delivers a design and experimental validation of the real-time time synchronisation characteristics achievable with IEEE 1588 . Several factors which can potentially affect the synchronisation accuracy in delivering a time reference from the master clocks to the end devices during both steady-state and transient changes were investigated. The objective is to increase the understanding of the real-time performance of a complete IEEE 1588 timing system applied in transmission substations, and identify the limitations of commercially-available devices.
\end{abstract}

Keywords—Global Navigation Satellite Systems, IEC 61850, IEEE 1588, protection and control, substation automation.

\section{INTRODUCTION}

Time synchronisation is essential to align the measurement processes associated with an event that happened in a power system; for example, it is needed to maintain the correct operation of wide area protection and control or other applications that utilise data from two or more measurement points. Phase measurement units (PMU), travelling wave fault locators, and more recently sample values (SVs) for use with the process bus in IEC 61850 based substations require time accuracy in the sub microsecond region. For instance, the IEEE C37.118 standard defines a maximum allowable Total Vector Error (TVE) for synchrophasors in PMU applications as 1\% which combines all error sources including magnitude, angle and time [1]. A 0.01 radian phase error will result in 1\% TVE by itself and lead to a time error of $31.8 \mu \mathrm{s}$ at $50 \mathrm{~Hz}$ or $26.5 \mu \mathrm{s}$ at $60 \mathrm{~Hz}$ systems. If only $20 \%$ error budget is assigned to time synchronisation, 5 to $6 \mu$ s timing precision is required [2]. The design goal for a fault location detector is a location precision of $200 \mathrm{~m}$ which translates to about $1 \mu \mathrm{s}$ in timing accuracy [3]. IEC 61850-9-2 Light Edition (9-2LE) also specifies $1 \mu$ s time stamping accuracy for synchronising Merging Units (MUs) [4].

The performance of power system applications due to inadequate timing accuracy have been widely studied. Authors in [5] investigated the effect of synchronisation errors on transformer differential protection. The impact of time deviation on synchrophasor-based wide area monitoring, protection and control (WAMPAC) was studied in [6]. The vulnerability of a WAMPAC system due to malicious time synchronisation spoofing attacks was analysed in [7].

For accurate timing in substations, Global Positioning System (GPS) receivers have been used since the early 1990's to obtain precise time information [8]. Manufacturers of GPS receivers for use in substations always claim an accuracy within $\pm 100 \mathrm{~ns}$, however, the performance of these receivers relies on the quality of the internal oscillator and the visibility of the satellites. The GPS signal power at a receiver is typically -160 $\mathrm{dBW}$; therefore, it is vulnerable to radio frequency interferences such as multipath due to reflections or diffractions experienced when the satellites are at low-elevation angles, or cyber-attacks caused by the deliberate or inadvertent jamming or spoofing of the satellite signals. To address this, other types of Global Navigation Satellite Systems (GNSS), i.e. GLONASS (Russia), Galileo (European Union) and BeiDou (China) are now being used in a single receiver to increase the overall satellite signal accuracy and availability [9].

1-PPS, the IRIG-B time code or more recently PTP are three options used for disseminating time with $1 \mu$ s precision to intelligent electronic devices (IEDs) [10]. 1-PPS and IRIG-B are well-established techniques, but require dedicated cables to distribute the time signals. A substation control room is often a few hundred meters away from outdoor cubicles in a large open-air substation. A large number of copper cables or optic fibres need to be deployed. 1-PPS and IRIG-B cannot automatically compensate the propagation delay; in copper and fibre cables this is approximately $5 \mathrm{~ns} / \mathrm{m}$ which results in a delay of $1 \mu \mathrm{s}$ for a $200 \mathrm{~m}$ cable [10]. The compensation of propagation delay demands considerable effort during installation and maintenance, especially in large substations.

IEEE 1588-2008, known as "PTPv2", is a networked timing method which achieves sub-microsecond accuracy with the aid of hardware time stamping techniques [11]. It uses bidirectional communication methods so the propagation delay is computed and compensated. Compared to other timing options, PTP 
offers benefits in terms of cost, security and accuracy; consequently, most newly-built substations are expected to use PTP for time synchronisation purposes [12] [13].

Various "profiles" have been introduced to allow PTP to be used across different engineering disciplines with minimum parameter settings. IEEE C37.238-2011 (Power Profile v1) was developed to satisfy the timing requirement of power system applications [14]. The first version was released in 2011, and it has now been revised and split into two standards: IEEE C37.238-2017 (Power Profile v2) [15] and IEC 61850-9-3: 2016 [16]. IEC 61850-9-3, which is called the "Base Profile", is the subset of IEEE 1588-2008 Annex F and Annex J.4 when clocks are singly attached. It also includes the redundant options for doubly attached clocks, which is compliant with IEC 62439-3:2016 Annex A [17]. Power Profile v2 extends the capabilities of IEC 61850-9-3 by providing additional features such as time inaccuracy reporting and extended support for IRIG-B converters.

This paper evaluates the real-time timing performance of IEEE 1588 for future transmission substations. The work extends the activities described in an earlier paper [18]. The main contributions of this paper are listed as follows: 1 . Designed and built a hardware testbed for assessing the realtime synchronisation performance of a combined GNSS and IEEE 1588 system. 2. Simulated various network scenarios using a network capture card, a traffic generator and a network impairment simulator. 3. Examined the real-time timing performance under the advanced IEC 61850 substation architectures. 4. Identified the characteristics of commerciallyavailable IEEE 1588 devices during steady-state and transient changes. 5. Provided the power industry with additional understanding of the synchronisation performance achievable with IEEE 1588.

The rest of the paper is organised as follows. Section 2 presents the theory behind IEEE 1588 . The experimental setup and the selected test methods are described in section 3, the results obtained are discussed in section 4 and the conclusions are presented in section 5 .

\section{IEEE 1588 SYNCHRONISATION PRINCIPLE}

IEEE 1588 establishes synchronisation based on a masterslave hierarchy. Slave clocks adjust the internal clocks to match with the master using the received synchronisation messages and include compensation of the propagation delay. Two delay measurement mechanisms are proposed in IEEE 1588-2008, namely End-to-End (E2E) and Peer-to-Peer (P2P), for measuring the propagation delay of the synchronisation messages [11].

E2E utilises the message exchange between the master and the slaves (Delay Request-Response Mechanism); the propagation delay is derived from the round trip time. However, the forwarding and receiving messages may not follow the same path due to system topology changes, which potentially affects the synchronisation accuracy. One the other hand, P2P utilises Peer Delay Mechanism; the path delay between each two adjacent devices is measured independently, hence the delay measurement message sent from slave to master is not needed.

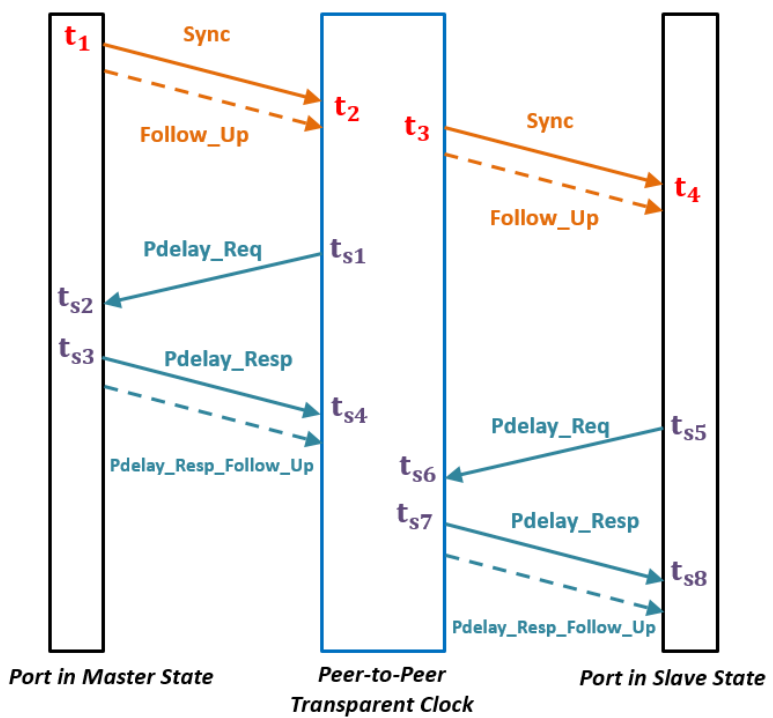

Fig. 1. Working principle of IEEE 1588 using peer-to-peer delay mechanism.

IEEE C37.238 and IEC 61850-9-3 requires the mandatory use of P2P mechanism for time synchronisation in substation automation systems [14] [15] [16].

Fig. 1 describes the working principle of IEEE 1588 using P2P. The messages shown are categorized into two types, event and general messages, as defined in IEEE 1588-2008 [11]. Sync, Pdelay_Req and Pdelay_Resp are event messages, which require accurate hardware timestamping. General messages comprise of Follow_Up, Pdelay_Resp_Follow_Up, which are only used to carry information so timestamping is not required.

The calculation of the path delay between the master and the Transparent Clock (TC) is described as follows:

- The TC sends a Pdelay_Req message to the master and timestamps the message $\left(t_{s 1}\right)$ on leaving the egress port.

- When it receives the Pdelay_Req, the master generates a timestamp $\mathrm{t}_{\mathrm{s} 2}$ and a Pdelay_Resp message is prepared.

- The master timestamps Pdelay_Resp $\left(\mathrm{t}_{\mathrm{s} 3}\right)$ when it departs; for one-step mode, the turnaround time $\left(\mathrm{t}_{\mathrm{s} 3}-\mathrm{t}_{\mathrm{s} 2}\right)$ is added to correction field of Pdelay_Resp, whilst for two-step. Pdelay_Resp_Follow_Up is applied.

- Once Pdelay_Resp reaches the TC, a timestamp $\mathrm{t}_{\mathrm{s} 4}$ is issued.

The path delay is calculated using (1) and the link delay between the slave and the TC, trink_ts, using (2)

$$
\begin{aligned}
& t_{\text {link_mt }}=\frac{\left(t_{s 4}-t_{s 1}\right)-\left(t_{s 3}-t_{s 2}\right)}{2} \\
& t_{\text {link_ts }}=\frac{\left(t_{s 8}-t_{s 5}\right)-\left(t_{s 7}-t_{s 6}\right)}{2}
\end{aligned}
$$

Based on the knowledge of peer delay, the propagation delay of the Sync message is measured as follows:

- When the Sync leaves the egress port of the master, a timestamp denoted as $t_{1}$ is issued and placed with Sync (one-step) or Follow_Up (two-step)

- When the Sync passes through the transparent clock, timestamp $t_{2}$ and $t_{3}$ are generated at the ingress and egress ports, respectively. The residence time $\left(t_{3}-t_{2}\right)$ together with the path delay, tlink_mt, are summed and added to the 
correction field ( $\mathrm{t}_{\text {correction}}$ ) of Sync, or Follow_Up in the twostep mode.

- When the slave receives the Sync message, it timestamps the incoming message at the ingress port as $t_{4}$. $t_{\text {link }} t_{s}$ is added to tcorrection of the Sync or Follow_Up (two-step)

The time offset between slave and master can thus be derived from (3) and (4). The slave clock adjusts its internal clocks based on toffset.

$$
\begin{aligned}
& \mathrm{t}_{\text {correction }}=\mathrm{t}_{\text {link_mt }}+\mathrm{t}_{\text {link_ts }}+\left(\mathrm{t}_{3}-\mathrm{t}_{2}\right) \\
& \mathrm{t}_{\text {offset }}=\mathrm{t}_{4}-\mathrm{t}_{1}-\mathrm{t}_{\text {correction }}
\end{aligned}
$$

\section{EXPERIMENTAL SETUP AND METHODOLOGY}

An IEEE 1588 testbed was built in accordance with the schematic diagram in Fig. 2. Grandmaster A was equipped with an oven controlled crystal oscillator (OCXO) with GPS constellation access. It provided the most stable timing signals of all three clocks. Grandmaster B and C were both fitted with a temperature compensated crystal oscillator (TCXO) and featured multi-constellation receivers, i.e. they could receive GPS and GLONASS signals. Two Slave clocks, $\mathrm{X}$ and $\mathrm{Y}$ were built by two different manufactures and were configured to operate in slave-only mode during the experiment. The Local Area Network (LAN) cloud represents different network topologies formed by PTP compatible Ethernet switches. Switch $\mathrm{H}$ and $\mathrm{M}$ were supplied by the same manufacture, but Switch $\mathrm{H}$ is the latest version. All switches could be configured as either two-step Transparent Clocks (TCs) or Boundary Clocks (BCs). They can also be used as Redundancy Boxes (Redboxes) during PRP or HSR implementations. A traffic generator (impairment emulator) was also included to inject SV traffic at different transmit rates or to create impairment for specific packets. This is particularly important for stressing the network and for evaluating the synchronisation limit. IEC 61850-9-3 profile was used as the benchmark for the configuration.

All the Ordinary Clocks (OCs) had a maximum $100 \mathrm{Mb} / \mathrm{s}$ bandwidth with copper 100BASE-TX connections. Switch M had four copper 100BASE-TX connections and six 100BASEFX optical interfaces. Switch $\mathrm{H}$ has the same port arrangement as Switch M, except for two optical interfaces which support 1000BASE-FX. In all cases, $1.5 \mathrm{~m}$ Cat 5e twisted pair Ethernet

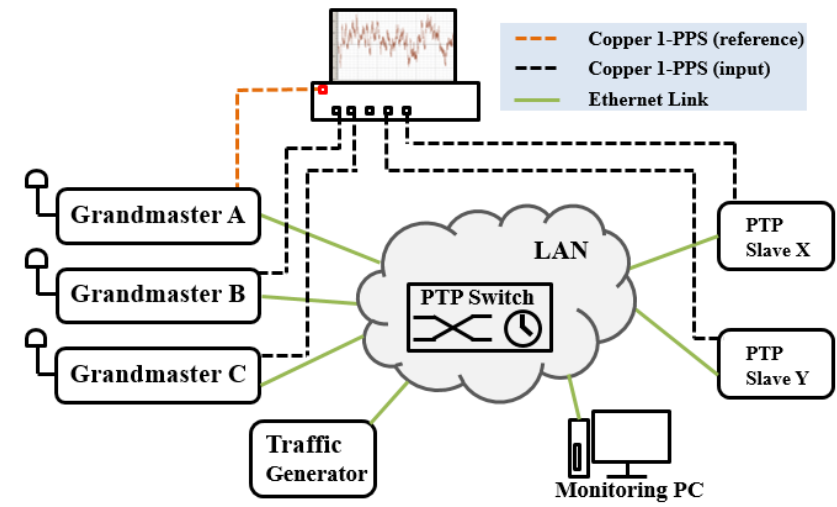

Fig. 2. Schematic diagram of system setup cables were used for connections between clocks and switches, and $5 \mathrm{~m}$ optical fibres with LC connectors were used between switches.

The synchronisation performance assessment described in the paper falls into two categories: steady-state (section 3.1 and 3.2) and transient (section 3.3 to 3.6). Each transient-state experiment was repeated many times and the results presented here is a typical example to illustrate the characteristics. The timing accuracy was evaluated by using a measurement server to compare the 1-PPS signal output between different clocks. All cables connected to the server were $1.5 \mathrm{~m} 50 \Omega$ RG58 coaxial with BNC connectors. The description and accuracy validation of the server are described in [18]. The measurement error associated with the server was correctly calibrated by modifying its configuration settings.

The experiment was performed at room temperature; this corresponds to a measured ambient temperature of $20{ }^{\circ} \mathrm{C}$ to $25{ }^{\circ} \mathrm{C}$. The temperature variation is considered acceptable and has minimal impact on synchronisation accuracy; this is because all the devices were equipped with either a TCXO or an OCXO.

\subsection{Long Term Satellite Visibility and Synchronisation Accuracy based on Different Constellation Selections}

The distance between a satellite and a receiver is obtained by measuring the signal delay. For achieving a global coverage, a minimum number of 4 satellites must be above the horizon at any time [19]. The greater the number of satellites seen by a receiver, the lower the possibilities of losing synchronisation and the higher the accuracy of positioning.

The experimental setup illustrated in Fig. 3 was used to first investigate the effect of constellation selection on satellite visibility. Three consecutive 24-hour measurements were carried out using the multi-GNSS receiver of Grandmaster B. It also acted as the grandmaster clock to synchronise Slave Y using PTP. The satellite status was monitored and the statistics were logged into the Monitoring PC.

The timing accuracy of receivers also depends on the algorithm they use to decode satellite signals. Although multiGNSS receivers claim to have higher satellite visibility and utilisation rate, this does not prove the accuracy of synchronisation is enhanced by having more satellites in view. Hence, as shown in Fig. 3, the 1-PPS output of Grandmaster B was connected to the measurement server as input. The reference 1-PPS was obtained from Grandmaster A, as it was equipped with a highly stable OCXO. The 1-PPS of slave $\mathrm{Y}$ was also measured against the reference. In all cases the antenna

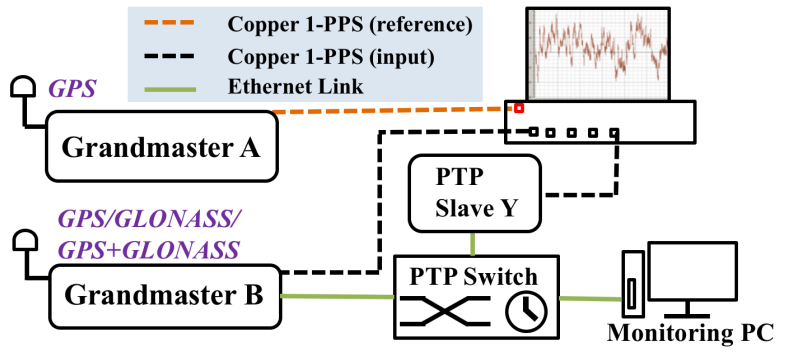

Fig. 3. Experimental setup to evaluate the effect of constellation selection on satellite visibility and timing accuracy. 


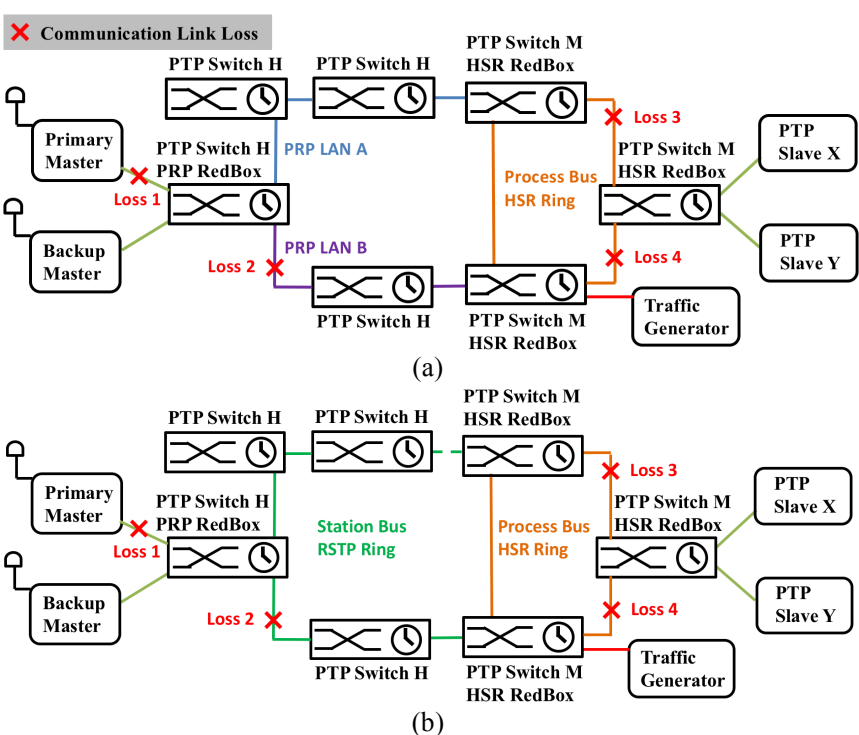

Fig. 4 Highly redundant network architecture: (a) PRP coupled HSR, (b) RSTP coupled HSR.

cable lengths and the propagation delays of 1-PPS signals were correctly compensated.

The experiment was started at 22:00, $23^{\text {rd }}$ June 2018 and finished at 22:00, 26 $6^{\text {th }}$ June 2018. In the first 24-hour period, the GPS constellation was selected, followed by GLONASS for the next 24 hours and finally GPS+GLONASS.

\subsection{Synchronisation Performance under Highly Redundant Ring Topologies}

IEC 61850-90-4 introduces more advanced topologies for connecting the station bus and process bus in large scale transmission substations [20]. The timing accuracy of PTP under PRP coupled HSR (PRP-HSR) and RSTP coupled HSR (RSTP-HSR) are now discussed, as shown in Fig. 4. The station bus and process bus can also be separated by interconnecting them using IEDs. However, this requires all IEDs to perform as either TCs or BCs, which is not yet supported by all vendors. PTP switch M with $100 \mathrm{Mb} / \mathrm{s}$ port interfaces were used for the process bus and PTP switch $\mathrm{H}$ with $1000 \mathrm{Mb} / \mathrm{s}$ interfaces were chosen for the station bus. Grandmaster A and B were located at the same station bus switch, and were selected as primary master and backup master, respectively. Slave $\mathrm{X}$ and $\mathrm{Y}$ were placed at the same process bus switch. In real substation systems, grandmaster clocks are often located at different locations and are connected to different switches. This increases reliability, but from an experimental perspective connecting them to the same location ensures a better comparison of the clock performance, which is especially important for the transient study described in section 3.6.

To investigate the impact of network traffic on timing accuracy, a traffic generator was connected to a process bus switch. It was configured to inject multicast $S V$ traffic with a fixed size of 134 bytes and a speed of $80 \mathrm{Mb} / \mathrm{s}$. The VLAN priority 4 was assigned to $S V$ as specified in 9-2LE.

One of the main differences in IEC 61850-9-3 as compared with Power Profile v1, is the removal of the mandatory use of VLANs [21]. The original intention in using the VLAN field in Power Profile v1 was to separate different applications so the

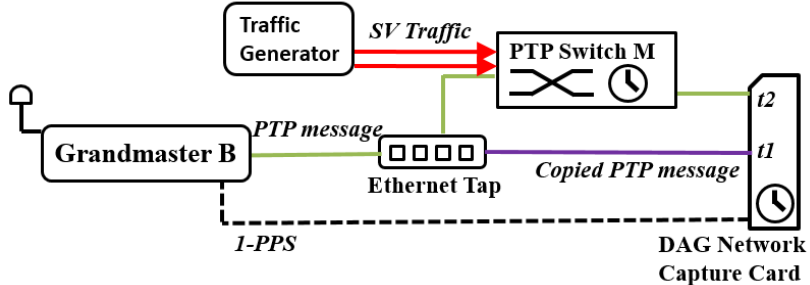

Fig. 5. Schematic overview of the experimental setup for investigation of the PTP message latency and loss rate under excessive SV traffic with the aid of the Ethernet tap and Capture card.

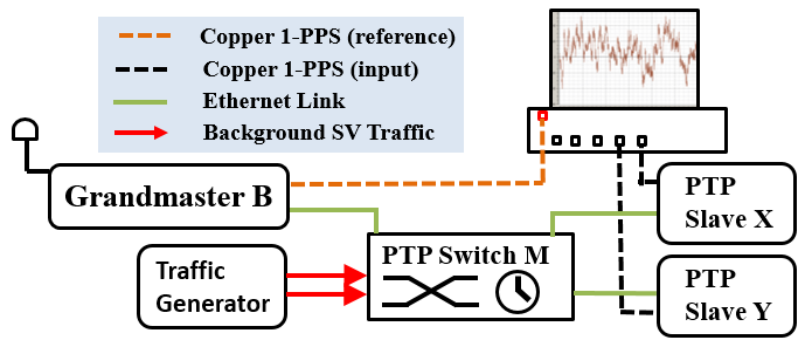

Fig. 6. Schematic overview of system setup for investigation of real-time slave clock synchronisation performance under excessive SV traffic.

security and dependability can be improved and the priority field was aimed to improve the performance of PTP messages in terms of transmission latency, jitter, and loss rate under heavy network traffic conditions [22]. Therefore, it is important to know if the synchronisation accuracy will be deteriorated by $S V$ traffic injection, when PTP loses the "protection" of VLAN priorities.

All the OCs were configured according to IEC 61850-9-3. Switches were configured as TCs. Each test was run for $1000 \mathrm{~s}$. Two network loading conditions were compared: No traffic and $80 \% S V$ traffic. The synchronisation accuracy for the slave clocks were assessed by comparing their 1-PPS against the reference 1-PPS (Grandmaster A).

\subsection{Synchronisation Capability under Excessive SV Traffic}

To reduce the traffic level, multicast or VLAN filtering is often applied to specific switch ports so only relevant messages are forwarded. However, excessive network traffic conditions may occur due to bad network design or misconfiguration and too much traffic is likely to cause a loss of packets or a significant increase in latency. IEC 61850-9-3 PTP messages do not contain a VLAN priority tag, which means packet transmission is more likely to be affected by high priority traffic in a congested network.

In this section, the transfer latency and packet loss rate of Sync or Follow_Up messages under excessive SV traffic were first investigated. Grandmaster B was configured to transmit PTP messages without VLAN tags in two-step operation mode; these messages were injected into an Ethernet tap as shown in Fig. 5. A network capture card installed in the host machine via a PICe slot was used for packets capture. The card obtained time of day information using Network Timing Protocol (NTP) from the host PC, and utilised 1-PPS from Grandmaster B for finely tuning its internal oscillator. The capture card could precisely timestamp the incoming packets with a resolution of $7.5 \mathrm{~ns}$. By using the capture card and the Ethernet tap, two network streams containing the same set of PTP messages but with different arrival times can be captured. The copied PTP 
message is captured directly, whilst the second stream is passed through the $S V$ loaded switch before being captured. The transfer latency was evaluated by calculating the difference between the timestamps $(t 2-t 1)$ for two PTP messages having the same sequence number.

A traffic generator injected three levels of background $S V$ traffic $(100 \mathrm{Mb} / \mathrm{s} .150 \mathrm{Mb} / \mathrm{s}$ and $200 \mathrm{Mb} / \mathrm{s})$ into Switch $\mathrm{M}$ and three $S V$ priorities were chosen $(4,6$, and 7$)$ to investigate if PTP transmission would be affected by $S V$ prioritisations. 1000 Sync and Follow_Up messages were logged for computing the transfer latency and the packet loss rate.

The real-time synchronisation accuracy of Slave clocks under excessive $S V$ traffic conditions is also examined in this section. The experimental setup is depicted in Fig. 6. Grandmaster B was configured to transmit two-step PTP messages (no VLANs) to slave clocks via switch M. $S V$ traffic was injected at $100 \mathrm{Mb} / \mathrm{s} .150 \mathrm{Mb} / \mathrm{s}$ and $200 \mathrm{Mb} / \mathrm{s}$ to be consistent with the previous test.

\subsection{Impact of PTP Traffic from Multiple Time Domains}

A PTP domain defines a set of PTP devices in a logical group where the OCs, TCs and BCs synchronise to other devices within the same domain. PTP v2 allows the existence of PTP devices belonging to multiple domains in the same network where the operation and time scale in different domains are independent [11]. The default domain number for Power Profile v1 is 0, whilst IEC 61850-9-3 and Power Profile v2 specify 93 and 254 as the domain number, respectively [15][16].

A PTP compatible switch can support multiple domain operations, and is able to process PTP messages and quickly forward them to the correct destination. PTP switches which cannot correctly handle PTP traffic, will result in a loss of PTP messages and affect the synchronisation accuracy. The previous study investigated the performance limit of a PTP network under excessive PTP traffic within a single time domain [18]. However, the capability of a TC to process message from multiple domains was not addressed.

The test setup is shown in Fig. 7. Grandmaster A was configured to transmit PTP message in domain 0 with default transmit rate (1 message/s for Sync, Announce and Pdelay Req), whilst Grandmaster B and C were configured to have PTP message in domain 254 and 93 respectively. However, to create excessive PTP traffic, the Sync transmit interval of Grandmaster $\mathrm{B}$ and $\mathrm{C}$ varied during the experiment. The available setting for Grandmaster $\mathrm{B}$ and $\mathrm{C}$ were $2^{\mathrm{x}}$ where $\mathrm{x}$ had range from -7 to 4 . Hence, the maximum Sync transmit rate of Grandmaster B and C was 128 Sync/s. Slave X and Y were configured to domain 0 so they only synchronised to

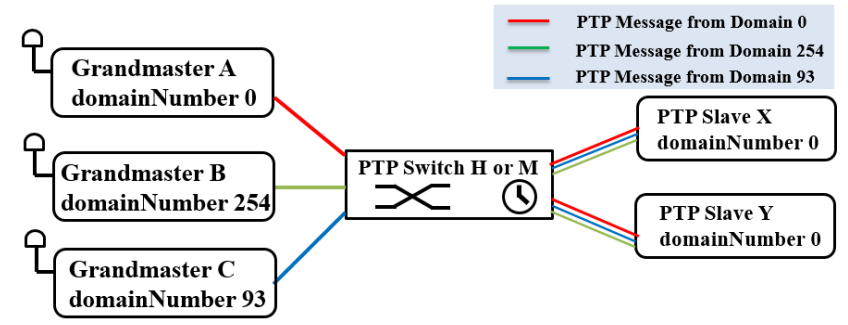

Fig. 7. Synchronisation performance under multi-domain PTP traffic.
Grandmaster A. The test was run for multiple repetitions following the same procedure. The Sync transmit rate of increased for every test repetition. Once the maximum transmit rate (128 Sync/s) of Grandmaster B was reached, Grandmaster $\mathrm{C}$ began to increase its Sync message rate. Switch $\mathrm{H}$ and $\mathrm{M}$ were both tested and their performance were compared.

\subsection{Slave Clock Characteristics during Follow_Up Message Delay}

Sync messages are timestamped when they enter or depart from a PTP device. The timestamp data is either encapsulated in the Sync message (one-step) or in a second message called the Follow_Up (two-step). Due to the network traffic or unexpected contingencies, Follow_Up may experience longer transfer time than its Sync pair. For example, if a 134 byte $S V$ frame is being transmitted in front of a Follow_Up message, it will result in approximately $10.72 \mu$ s delay at $100 \mathrm{Mb} / \mathrm{s}$ bandwidth. Likewise, a 1530 byte frame will result in approximately $122 \mu$ s extra delay. In a large substation with dozens of hops, the arrival time difference between a Sync and its Follow_Up message can be a few milliseconds in the worst case scenario. Hence, the experiments in this section investigate the transient behaviours of slave clocks if the Follow_Up arrives significantly later than its Sync message pair.

As shown in Fig. 8, a traffic impairment emulator was deployed to filter out the Follow_Up packets from Grandmaster A. Time delays were then deliberately added to all Follow_Up messages and the rest of the packets were unimpaired. All packets were merged and sent from the other port. The delay insertion was increased in steps of $5 \mathrm{~ms}$. Note: The product specification states the error of the inserted delay was within \pm 2560 ns, hence the errors caused by the emulator can be neglected.

During this test only Follow_Up impairment in the two-step operation mode was considered. Deliberate insertion of time delay on Sync messages in two-step mode will make Follow_Up messages arrive early than their Sync pair which violates the principle of peer delay measurement and causes loss of synchronisation. Insertion of time delay in one-step Sync messages will directly result in synchronisation errors as the Sync latency caused by the emulator is invisible to peer delay measurement so the delay cannot be measured precisely.

\subsection{Fault Tolerance Assessment of Highly Redundant System Architecture Due to Communication Link Loss}

The reliability of a timing system is important since it must ensure all time critical protection and control applications operate correctly. In this section, the transient characteristics of slave clocks on the communication link loss are discussed. Two architectures, PRP coupled HSR and RSTP coupled HSR were

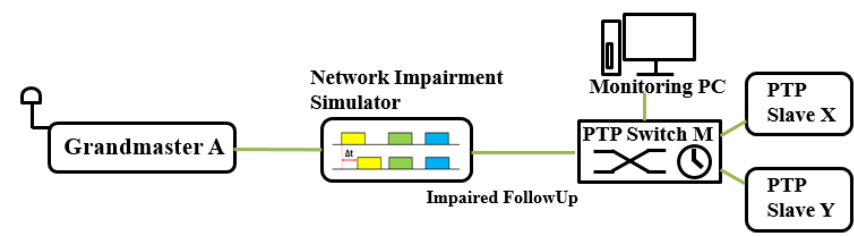

Fig. 8. Investigation on the impact of Follow_Up Delay on PTP synchronisation performance 
assessed as illustrated in Fig. 4. Grandmaster B and C used the GPS+GLONASS constellation, and were configured as the primary and backup master, respectively. The experimental procedure was as follows: Slave clocks synchronised to the primary master (Grandmaster B) for $200 \mathrm{~s}$; communication link between Grandmaster B and Switch $\mathrm{H}$ was immediately disconnected (Loss 1); a communication link on the station bus was then disconnected at $500 \mathrm{~s}$ (Loss 2); next, a communication link on the process bus was disconnected at $800 \mathrm{~s}$ (Loss 3$)$; then, a communication link operating in the opposite direction on the process bus was disconnected at $1100 \mathrm{~s}$ (Loss 4); and finally, all communication links were restored at $1500 \mathrm{~s}$.

\section{Results AND Discussions}

\subsection{Long Term Satellite Visibility and synchronisation Accuracy based on Different Constellation Selections}

Satellite visibility for GPS, GLONASS and GNSS during a 24-hour continuous measurement window is given in Fig. 9 (a). It is observed that at least 4 satellites were utilised at all time when GPS or GPS+GLONASS (GNSS) constellations were selected. GLONASS had poorer visibility and sometimes the number of used satellite dropped below 4 . An extreme situation occurred at 08:07, $23^{\text {rd }}$ June 2018 when information from only two GLONASS satellites were used by the receiver for approximately 30 seconds. The use of GNSS constellation significantly improved the overall satellite visibility by using more constellations. No less than 10 satellites were always available and used, and the number was roughly the summation of GPS and GLONASS, which satisfied our expectation.

The Satellite Trajectory plots for Grandmaster B under three constellation selections are illustrated in Fig. 10. The hole in the north is because satellites do no orbit over the North Pole. GLONASS had smaller hole than GPS which supports the argument GLONASS has a better coverage than GPS at higher

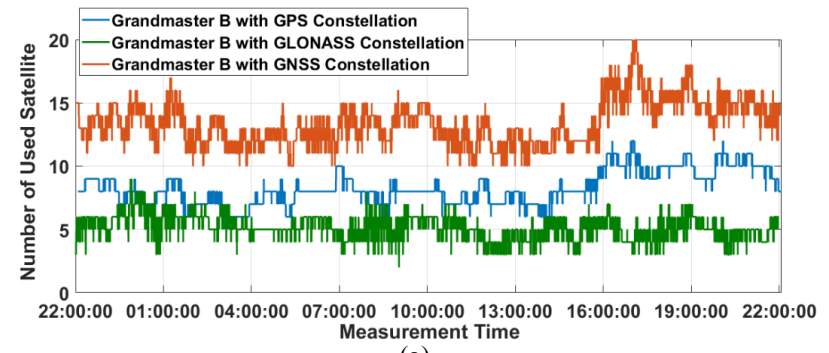

(a)

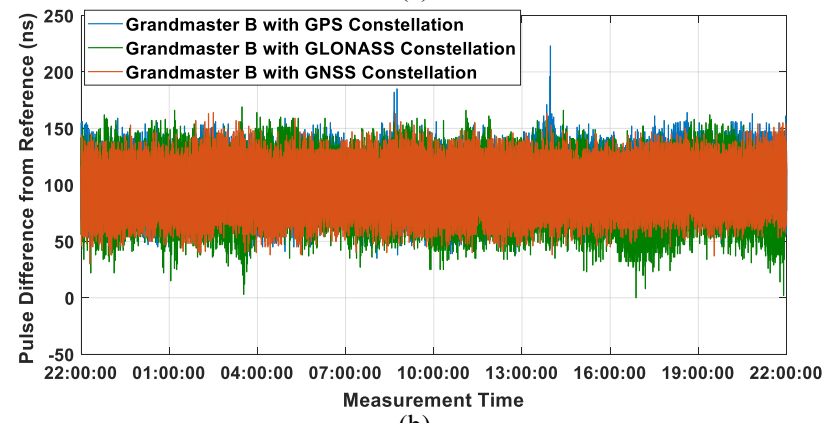

(b)

Fig. 9. Long term performance of satellite receivers of (a) satellite visibility and (b) synchronisation accuracy based on different constellation selections (with reference to Grandmaster A). latitudes [9]. However, a notch was found missing on the GLONASS plot, which was probably caused by a tall building located on the northeast side of the antenna (Fig. 11). This resulted in a degradation in the signal strength, which prevented satellites in this direction being observed by the receiver. Although GPS satellites provided less coverage than GLONASS in northern latitudes, the overall signal strength was stronger, and consequently, GPS visibility was not significantly obstructed by the tall building. GNSS had the best coverage, as it absorbed the advantages of both GPS and GLONASS.

The time series 1-PPS offset based on three constellations are plotted in Fig. 9 (b) and more detailed statistics are summarised in Table I. During the tests it was noticed a fixed 1-PPS offset existed between Grandmaster A (reference) and Grandmaster B. This offset is believed to be related to different internal oscillator designs, giving the fact that the cable delay was correctly compensated. Note: similar phenomenon was also observed in paper [23]. In addition, although the number of used GLONASS satellites was less than 4 for extended periods during the 24 hour cycle, the receiver was able to hold synchronisation. Compared with individual GPS and GLONASS constellations, GNSS delivered the most stable 1PPS output with a standard deviation of $16.928 \mathrm{~ns}$. Regarding the mean offset, GLONASS presented a 93.867 ns difference relative to the reference 1-PPS. GPS showed the largest offset at $100.982 \mathrm{~ns}$. As mentioned in section 3.1, the timing accuracy of receivers depends on the algorithm used to decode satellite signals. The results indicate Grandmaster B presented a deviation in timing for different constellations but the overall difference was small (less than $10 \mathrm{~ns}$ ).

Two 1-PPS spikes (08:45 and 13:57) were observed in the GPS constellation; but no correlation was found between the

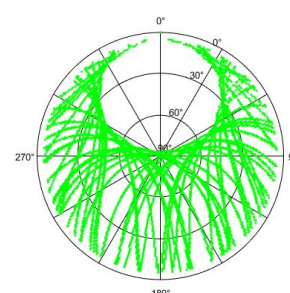

(a)

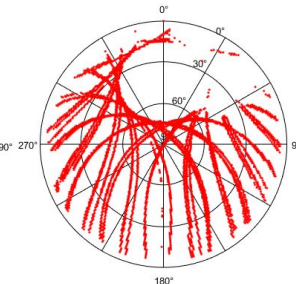

(b)

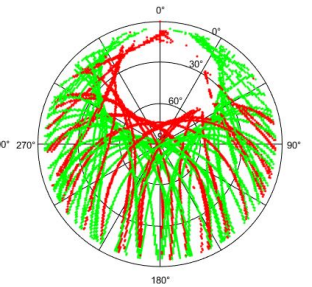

(c)
Fig. 10 Satellite trajectory plot on different constellation selections (a) GPS, (b) GLONASS, (c) GPS+GLONASS.

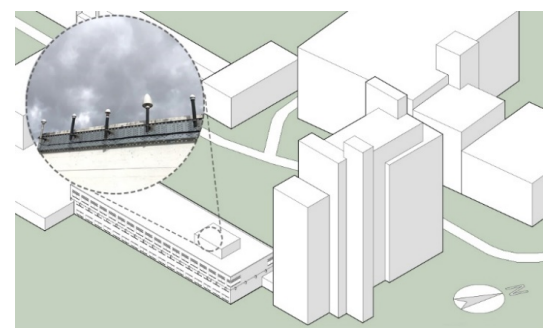

Fig. 11. Schematic diagram showing antenna locations with a tall building to the northeast

TABLE I

24-hour synchronisation accuracy of Grandmaster B under different constellations (with reference to Grandmaster A)

\begin{tabular}{cccc}
\hline \hline Constellation & $\bar{\alpha}(\mathrm{ns})$ & $\sigma(\mathrm{ns})$ & Range $(\mathrm{ns})$ \\
\hline GPS & 100.982 & 17.094 & 35 to 223 \\
GLONASS & 93.867 & 19.964 & 0 to 169 \\
GNSS & 96.967 & 16.928 & 30 to 164 \\
\hline \hline
\end{tabular}




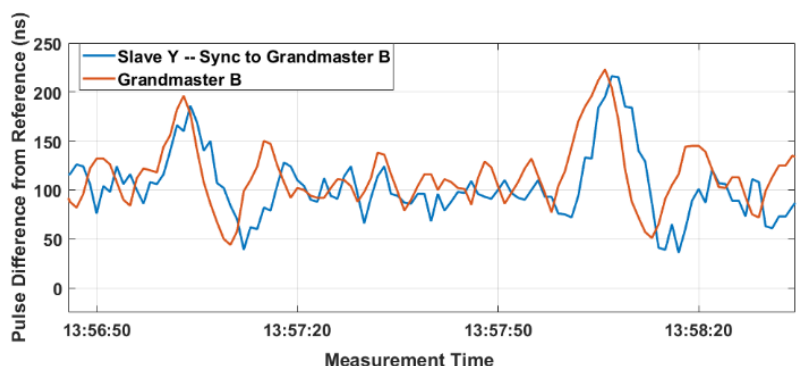

Fig. 12. 1-PPS offset of grandmaster $B$ and slave $Y$ (synchronised to grandmaster B via PTP) in comparison with reference 1-PPS.

spikes and the satellite conditions when the spikes occurred; i.e. there was no sudden change in the number of satellites or the signal strength at these periods. In addition, it was also confirmed that these spikes were not caused by deviations in the reference 1-PPS. Fig. 12 shows the 1-PPS offset of Grandmaster B and Slave Y in comparison with the reference. Slave Y was synchronised to Grandmaster B, i.e. its 1-PPS was tracking the change of Grandmaster B but as expected a small time delay was observed. If this offset was due to a sudden change in the accuracy of the reference, the spikes should be perfectly time aligned with each other. Therefore, the spikes observed in grandmaster B was more likely to be caused by multipath propagation resulting from signals received from satellites at low-elevation angle [9].

\subsection{Synchronisation Performance Under highly Redundant Ring Topologies}

The synchronisation performance for both slave $\mathrm{X}$ and $\mathrm{Y}$ under PRP-HSR and RSTP-HSR is summarised in Table II. Both systems achieved an accuracy level within $\pm 1 \mu \mathrm{s}$. RSTPHSR delivered a smaller mean offset compared with PRP-HSR, although their standard deviations were similar. In addition, 80 $\mathrm{Mb} / \mathrm{s}$ multicast $S V$ traffic had no influence on PTP synchronisation and no significant accuracy difference was found between slave $\mathrm{X}$ and $\mathrm{Y}$.

\subsection{Synchronisation Capability under Excessive SV Traffic}

The transfer latency and packet loss rate of Sync and Follow_Up messages under various traffic levels and $S V$ prioritisations are listed in Table III; as a benchmark, the test cases without traffic injection are also included. When the $S V$ priority was set to 4 or 6 , it was observed neither the Sync nor Follow_Up messages were affected by excessive $S V$ traffic. The Sync messages presented about $2 \mathrm{~ms}$ in terms of mean latency, which is approximately $2 \mathrm{~ms}$ less than the Follow_Up messages. The PTP switches used in this experiment were all
TABLE II

Synchronisation Performance of Slave X and Y using highly redundant ring topologies under various traffic conditions

\begin{tabular}{ccccc}
\hline \hline Topology, Traffic & Slave & $\bar{\alpha}(\mathrm{ns})$ & $\sigma(\mathrm{ns})$ & Range $(\mathrm{ns})$ \\
\hline PRP-HSR & $\mathrm{X}$ & 66.056 & 34.033 & -84 to 176 \\
& $\mathrm{Y}$ & 62.088 & 40.427 & -84 to 184 \\
PRP-HSR & $\mathrm{X}$ & 64.176 & 32.025 & -32 to 172 \\
$80 \mathrm{Mb} / \mathrm{s}$ & $\mathrm{Y}$ & 62.532 & 37.285 & -48 to 172 \\
& $\mathrm{X}$ & 11.364 & 35.906 & -88 to 104 \\
RSTP-HSR & $\mathrm{Y}$ & 8.195 & 37.932 & -108 to 116 \\
& $\mathrm{X}$ & 17.744 & 31.070 & -76 to 116 \\
RSTP-HSR & $\mathrm{Y}$ & 13.132 & 32.842 & -93 to 144 \\
$80 \mathrm{Mb} / \mathrm{s}$ & &
\end{tabular}

two-step TCs in which PTP packets forwarding were achieved in software for manipulating the content. Therefore, PTP transfer time were significantly longer than other Ethernet messages such as $S V$ and GOOSE (in microseconds). Packets were only dropped when $S V$ traffic with priority 7 approached the wire speed $(100 \mathrm{Mb} / \mathrm{s})$, and the transmission latency of both Sync and Follow_Up were significantly prolonged to more than $500 \mathrm{~ms} .24 \%$ of the Sync messages were lost at $100 \mathrm{Mb} / \mathrm{s}$ traffic, which then increased with traffic injection and reached $59 \%$ at $200 \mathrm{Mb} / \mathrm{s}$. Similarly, Follow_Up messages were also dropped under excessive traffic with priority 7 , but the packet loss rate were much higher than Sync at the same traffic level.

This was an interesting observation, as the VLAN field for the PTP messages were disabled in Grandmaster B. According to the manufacture specification, inbound packets without

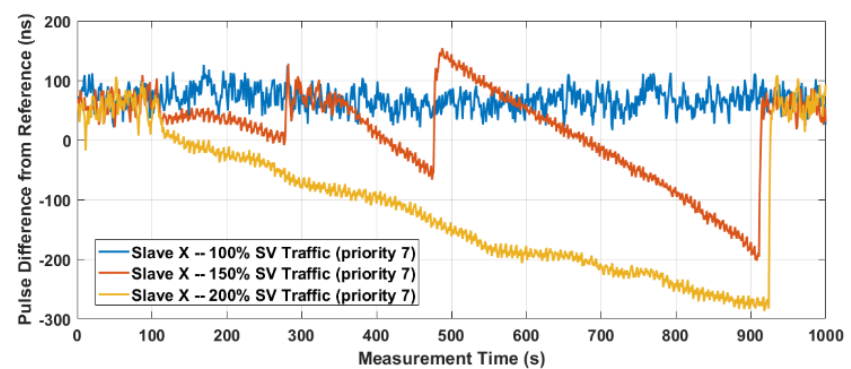

(a)

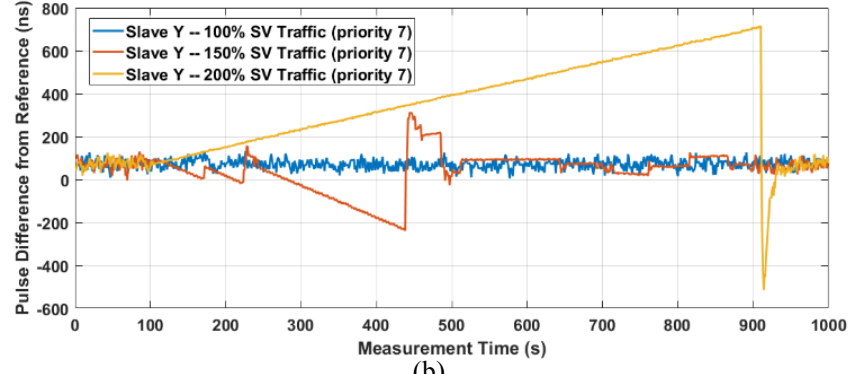

(b)

Fig. 13. Transient behaviours of (a) Slave $X$ and (b) Slave $Y$ under excessive SV traffic (with reference to Grandmaster B)

TABLE III

Sync and Follow Up message transfer latency and packet loss rate under excessive SV traffic

\begin{tabular}{|c|c|c|c|c|c|c|c|}
\hline \multirow[b]{2}{*}{ Traffic Level } & \multirow[b]{2}{*}{ Priority } & \multicolumn{3}{|c|}{ Sync Transfer Latency } & \multicolumn{3}{|c|}{ Follow_Up Transfer Latency } \\
\hline & & Mean (ms) & Range (ms) & Loss Rate & Mean $(\mathrm{ms})$ & Range (ms) & Loss Rate \\
\hline \multirow[t]{2}{*}{ No Traffic } & N/A & 2.045 & 1.840 to 5.618 & $0 \%$ & 4.022 & 3.582 to 8.719 & $0 \%$ \\
\hline & 4 & 2.115 & 1.879 to 5.937 & $0 \%$ & 4.013 & 3.622 to 8.755 & $0 \%$ \\
\hline \multirow[t]{3}{*}{$100 \%$ Traffic } & 6 & 2.088 & 1.884 to 5.961 & $0 \%$ & 3.995 & 3.625 to 9.214 & $0 \%$ \\
\hline & 7 & 536.167 & 470.740 to 590.121 & $24 \%$ & 542.246 & 480.727 to 599.191 & $73 \%$ \\
\hline & 4 & 2.124 & 1.887 to 6.057 & $0 \%$ & 4.017 & 3.625 to 8.719 & $0 \%$ \\
\hline \multirow[t]{3}{*}{$150 \%$ Traffic } & 6 & 2.079 & 1.880 to 6.085 & $0 \%$ & 3.966 & 3.615 to 7.811 & $0 \%$ \\
\hline & 7 & 513.714 & 454.119 to 592.282 & $50 \%$ & 517.808 & 467.48 to 598.24 & $86 \%$ \\
\hline & 4 & 2.122 & 1.872 to 4.55 & $0 \%$ & 4.040 & 3.607 to 7.269 & $0 \%$ \\
\hline \multirow[t]{2}{*}{$200 \%$ Traffic } & 6 & 2.101 & 1.880 to 5.192 & $0 \%$ & 4.003 & 3.611 to 7.764 & $0 \%$ \\
\hline & 7 & 523.531 & 471.89 to 562.694 & $59 \%$ & 531.697 & 487.952 to 570.14 & $93 \%$ \\
\hline
\end{tabular}


VLAN were treated with priority 0 (Best Effort) by Switch M during switching. Hence, in theory, some Sync and Follow_Up messages will experience additional transfer delay during traffic injection, and some PTP messages will be dropped when the traffic level is above the link bandwidth as the buffer in the switch is occupied by $S V$. However, this situation only happened when the $S V$ priority was set to 7 . The previous study [18] found the IEEE 1588 timing system was not affected by excessive background $S V$ traffic. The results in this section indicate that regardless of the VLAN settings PTP messages are treated with the highest priority by the chosen PTP switch. This is not defined in any profiles or standards and could be a vendor-specific implementation.

The real-time synchronisation performance of slave clocks under excessive $S V$ traffic with priority 7 is shown in Fig. 13. The results show both slave clocks maintained synchronisation at $100 \mathrm{Mb} / \mathrm{s} S V$ traffic even though some PTP messages were lost. It also indicates the PTP switch accurately measured the Sync latency even though the latency significantly increased. When $150 \mathrm{Mb} / \mathrm{s}$ traffic was injected, loss of synchronisation occurred and the slave clocks began to wander. However, they periodically dragged the delay offset back to a smaller value (as the zigzag shape shown on the figure). This is because some of the Sync/Follow_Up pairs were not dropped and the slave clocks could use them to regain synchronisation. Whereas, synchronisation was completely lost on both slave clocks with $200 \mathrm{Mb} / \mathrm{s}$ traffic injection. The results prove slave clocks had tolerance regarding PTP packet loss, and could hold synchronisation under some extreme traffic conditions. Slave clocks presented different synchronisation characteristics which is due to the design of their internal oscillators, and control algorithms [24].

\subsection{Impact of PTP Traffic from Different Time Domains}

The synchronisation accuracy of slave X and Y under PTP traffic from multiple time domains is shown in Fig. 14. With Switch M, Slave X started to wander when 192 Sync/s messages from other time domains (128 from domain 254 and 64 from domain 93) were loaded into the network, as indicated in Fig. 14, from $100 \mathrm{~s}$ onwards. Slave $\mathrm{Y}$ was able to hold synchronisation for an additional $120 \mathrm{~s}$ before it also started to wander. Both clocks regained synchronisation to Grandmaster A when multi-domain PTP traffic injection ceased at $800 \mathrm{~s}$.

The capacity to accommodate multi-domain PTP messages

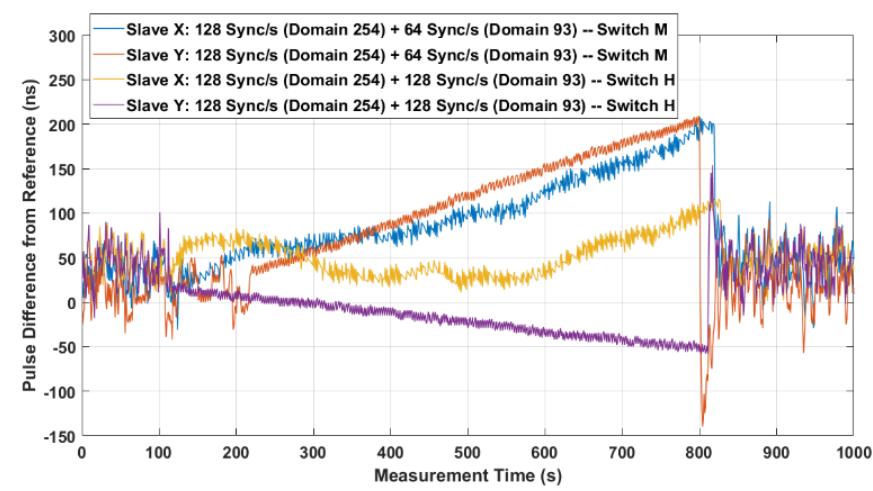

Fig. 14. Impact of multi-domain PTP traffic on synchronisation performance (with reference to Grandmaster A). was slightly improved with switch $\mathrm{H}$. Synchronisation was lost when 256 Sync/s (128 from domain 254 and 128 from domain 93) were injected. Although, 256 Sync/s from other time domains is unlikely to happen in normal conditions, it is important to pay attention to misconfiguration (some clock manufacturers offer considerable flexibility in configuring the PTP transmit interval) or cyber-attacks.

\subsection{Slave Clock Characteristics during Follow_Up Message Delay}

The effect of Follow_Up delay on PTP timing for both Slave $\mathrm{X}$ and $\mathrm{Y}$ are illustrated in Fig. 15 (a) and (b) respectively. When the Sync transmit rate was one message per second, Slave X lost synchronisation when the Follow_Up delay reached $1005 \mathrm{~ms}$. However, synchronisation loss of Slave Y occurred at a $405 \mathrm{~ms}$ Follow_Up delay. In comparison, at 2 Sync/s, Slave X started to wander when the delay reached $505 \mathrm{~ms}$, whilst slave $\mathrm{Y}$ again lost synchronisation at $405 \mathrm{~ms}$. Both slave clocks were desynchronised when a $205 \mathrm{~ms}$ delay was added to Follow_Up at 4 Sync/s.

A pattern was observed for Slave X; if the Sync interval was less than the delay being added to the follow up messages, synchronisation loss would occur. This is because slave $\mathrm{X}$ erased the "old" Sync message after the new one arrived. Therefore, when the impaired Follow_Up message arrived, it did not match the Sync message sequence number and was dropped. In contrast, Slave Y lost synchronisation when the Follow_Up delay impairment exceeded $400 \mathrm{~ms}$ during 1 Sync/s and 2 Sync/s conditions. This infers that Slave Y only preserved the Sync messages for a maximum time of $400 \mathrm{~ms}$. Results indicated both slave clocks did not store the previously arrived Sync messages. Therefore, attention should be raised for two-step operation mode, especially when a network contains many hops or is extremely congested, which results in a significant delay for the Follow_Up messages.

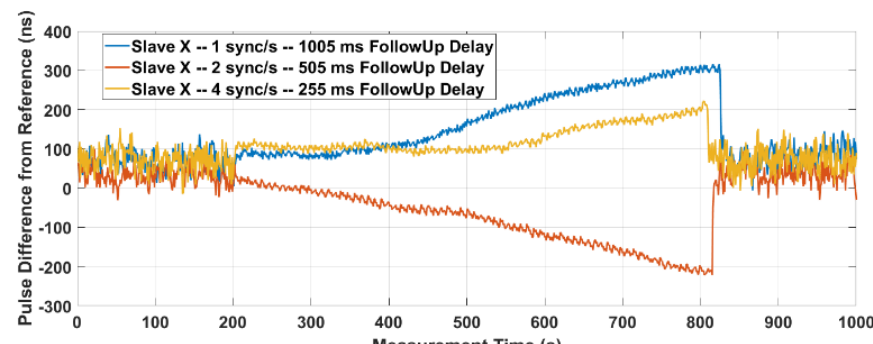

(a)

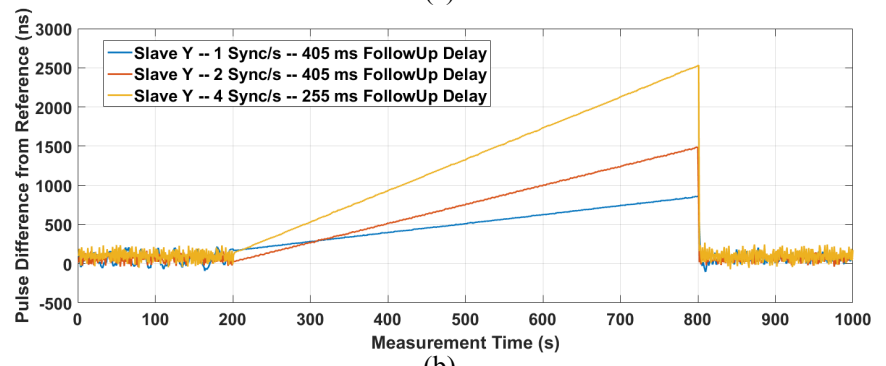

(b)

Fig. 15. Synchronisation performance of (a) Slave X and (b) Slave Y under Follow_Up delay impairment (with reference to Grandmaster A). 


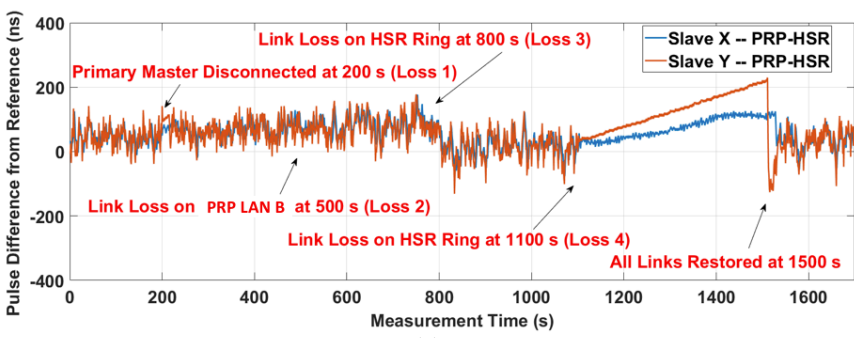

(a)

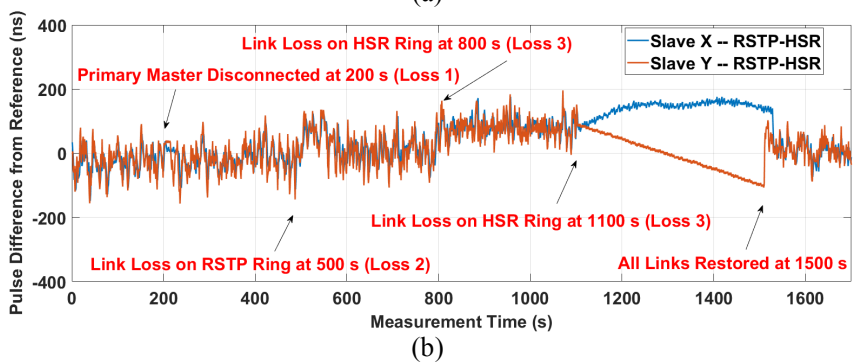

Fig. 16. Fault tolerance of (a) PRP-HSR and (b) RSTP-HSR due to communication link loss (with reference to Grandmaster B)

\subsection{Fault Tolerance Assessment of Highly Redundant System} Architecture Due to Communication Link Loss

Fig. 16 illustrates what happened during multiple communication link losses in highly redundant network topologies. It can be seen the BMCA transition at $200 \mathrm{~s}$ was smooth for both network topologies (Loss 1). When a single communication loss occurred in the station bus at 500s (Loss 2), PRP-HSR was able to hold the synchronisation smoothly, whilst RSTP-HSR presented a small "glitch" with a magnitude of around 50 ns. This was probably caused by incorrect calculation of some PTP messages latency during RSTP ring reconfiguration. Both networks were then able to maintain synchronisation during the loss of one communication link in the process bus HSR ring at $800 \mathrm{~s}$ (Loss 3 ). However, a $+50 \mathrm{~ns}$ shift in 1-PPS was observed with RSTP-HSR and a -50 ns with PRP-HSR. When the second link loss occurred in the process bus ring (Loss 4), all slave clocks could not receive the PTP messages from a master clock and started free-running. Finally synchronisation was regained when the communication links were restored.

\section{Conclusion}

This paper provided a design and testing guidance for using GNSS and IEEE 1588 time synchronisation systems in a transmission substation. Presented results have shown the multi-GNSS receiver combines the advantages of different constellation systems and offers a better satellite visibility and more reliable timing output. This is particularly important for satellite antennas with obstructed view of sky. PTP could meet the stringent $\pm 1 \mu$ s accuracy requirement with a significant margin under highly redundant substation architectures. It was identified that PTP messages were treated with the highest priority by the switches used in the test. In addition, although the operation of PTP in different domains are considered to be independent, the capability of a TC to process PTP messages is limited. Hence, synchronisation in one time domain can be affected by excessive PTP traffic from other domains. Delayed arrival of Follow $U p$ in the two-step operation mode would result in degradation of the timing accuracy if Follow_Up arrived significantly later than their Sync message pair. PRPHSR and RSTP-HSR can cope with "N-3" contingencies without losing synchronisation.

The work presented here adds additional knowledge to IEEE 1588 synchronisation network operation, and provides a better understanding on its performance limitations. PTP is gradually gaining acceptance in IEC 61850 process bus applications and component-level tests such as those described in this paper are necessary to ensure the power industry is confident in applying this technology in future substations.

\section{ACKNOWLEDGMENT}

The authors acknowledge financial support from EPSRC (EP/L016141/1) through the Power Networks Centre for Doctoral Training.

\section{REFERENCES}

[1] IEEE Standard for Synchrophasor Measurements for Power Systems, IEEE Std C37.118.1-2011, Dec. 2011.

[2] A. Carta, N. Locci, C. Muscas, F. Pinna, and S. Sulis, "GPS and IEEE 1588 synchronization for the measurement of synchrophasors in electric power systems," Comput. Stand. Interfaces, vol. 33, no. 2, pp. 176-181, Feb. 2011.

[3] A. Elhaffar and M. Lehtonen, "An improved GPS current traveling-wave fault locator in EHV transmission networks using few recordings," in 2005 Int. Conf. Futur. Power Syst., 2005, pp.1-5.

[4] Communication Networks and Systems for Power Utility AutomationPart 9-2: Specific Communication Service Mapping (SCSM)—Sampled Values Over ISO/IEC 8802-3, IEC 61850-9-2 ed2.0, Sep. 2011, IEC TC57.

[5] D. M. E. Ingram, P. Schaub, R. R. Taylor, and D. A. Campbell, "SystemLevel Tests of Transformer Differential Protection Using an IEC 61850 Process Bus," IEEE Trans. Power Deliv., vol. 29, no. 3, pp. 1382-1389, Jun. 2014.

[6] T. Bi, J. Guo, K. Xu, L. Zhang, and Q. Yang, "The Impact of Time Synchronization Deviation on the Performance of Synchrophasor Measurements and Wide Area Damping Control," IEEE Trans. Smart Grid, vol. 8, no. 4, pp. 1545-1552, 2017.

[7] M. S. Almas, L. Vanfretti, R. S. Singh, and G. M. Jonsdottir, "Vulnerability of Synchrophasor-based WAMPAC Applications' to Time Synchronization Spoofing," IEEE Trans. Smart Grid, vol. 9, no. 5, pp. 11, Sep. 2018.

[8] Wen An et al., "A transmission utility's experience to date with feeder unit protection systems," 11th IET Int. Conf. Dev. Power Syst. Prot. (DPSP 2012), pp. 31-31, Apr. 2012.

[9] L. Heng, T. Walter, P. Enge, and G. X. Gao, "GNSS Multipath and Jamming Mitigation Using High-Mask-Angle Antennas and Multiple Constellations," IEEE Trans. Intell. Transp. Syst., vol. 16, no. 2, pp. 741750, Apr. 2015

[10] D. Ingram and B. Smellie, "Solving Electrical Sbstation Timing Problems-A white paper on the use of the Precision Time Protocol for substation protection and control systems," 2014.

[11] IEEE Std 1588-2008, IEEE Standard for a Precision Clock Synchronization Protocol for Networked Measurement and Control Systems, IEEE Std 1588-2008, Jul. 24, 2008.

[12] K. Hinkley and C. Mistry, "First Digital Substation in TransGrid Australia: A journey,Business case, Lessons," J. Eng., Jul. 2018.

[13] J. Zhao et al., "A network scheme for process bus in smart substations without using external synchronization," Int. J. Electr. Power Energy Syst., vol. 64, pp. 579-587, 2015.

[14] IEEE Standard Profile for Use of IEEE 1588 Precision Time Protocol in Power System Applications, IEEE Std C37.238-2011, Jul. 14, 2011.

[15] IEEE Standard Profile for Use of IEEE 1588 Precision Time Protocol in Power System Applications, IEEE Std C37.238-2017, Jun. 2017.

[16] IEC/IEEE International Standard - Communication networks and systems for power utility automation Part 9-3: Precision time protocol profile for 
power utility automation, IEC/IEEE 61850-9-3, May. 2016.

[17] IEC 62439-3:2016, Industrial communication networks - High availability automation networks - Part 3: Parallel Redundancy Protocol (PRP) and High availability Seamless Redundancy (HSR). .

[18] H. Guo and P. Crossley, "Design of a Time Synchronization System Based on GPS and IEEE 1588 for Transmission Substations," IEEE Trans. Power Deliv., vol. 32, no. 4, pp. 2091-2100, 2017.

[19] J. E. Draim, "Tetrahedral multi-satellite continuous-coverage constellation." Google Patents, 1989.

[20] Communication networks and systems for power utility automation. Network engineering guidelines, IEC/TR 61850-90-4:2013, Aug. 06, 2013.

[21] M. Adamiak et al., "Revision of IEEE Std C37. 238, Power Profile for IEEE-1588: Why The Big Changes?," in Georgia Tech Prot. Relaying Conf. 2018, 2018.

[22] G. S. Antonova et al., "Standard profile for use of IEEE Std 1588-2008 Precision Time Protocol (PTP) in power system applications: IEEE PES PSRC Working Group H7/Sub C7 members and guests," in 2012 IEEE Int. Symp. Precis. Clock Synchronization Meas. Control Commun. Proc., 2012, pp. 1-6.

[23] D. M. E. Ingram, P. Schaub, D. A. Campbell, and R. R. Taylor, "Quantitative assessment of fault tolerant precision timing for electricity substations," IEEE Trans. Instrum. Meas., vol. 62, no. 10, pp. 2694-2703, 2013.

[24] W. Han, X. Shen, E. Hou, and J. Xu, "Precision time synchronization control method for smart grid based on wolf colony algorithm," Int. J. Electr. Power Energy Syst., vol. 78, pp. 816-822, Jun. 2016. 